\title{
Pricing of delivery services in the e-commerce sector
}

\author{
Claire Borsenberger ${ }^{*}$, Helmuth Cremer $^{\dagger}$, Philippe De Donder, \\ Denis Joram ${ }^{\S}$ and Sébastien Lécou ${ }^{* *}$
}

Electronic commerce (e-commerce) is an expanding activity, even though currently it still represents a rather small share of total retail commerce (4-7\% according to the estimates in France, and around 4\% in Europe). In 2012, in France, online sales reached $€ 45$ billion (Fevad, 2013), which represents a 19\% increase in a single year. By comparison, overall retail and commercial craft sales grew by "only" $3.1 \%$ in value in 2011 .

E-commerce poses many interesting questions for economists. The existing literature has concentrated on its drivers at a macro- and micro-level (Javalgi and Ramsey, 2001; Brousseau, 2003), on its specificities compared with traditional brick-and-mortar commerce, on issues related to trust and sellers reputation (Cabral and Hortaçsu, 2010), on the competition between these forms of commerce (Clay et al., 2002), on the price competitiveness of e-commerce (Brynjolfsson et al., 2003). However, the vertical relationships in the e-commerce sector between e-retailers and their inputs' providers, like the delivery services, and the optimal pricing strategies of the latter have received little attention so far. Related subjects, like the pricing of publicly produced inputs, have been dealt with (Feldstein, 1972 Spencer and Brander, 1983).

A comprehensive model of the e-commerce sector would account for the competition between e-commerce firms while also including the market for delivery services that constitutes an important input into the production function of e-retailers. From the delivery operator's perspective, the competition between e-commerce firms is important because it determines to what extent delivery rates are passed through to consumers, which in turn determines the final demand for products as well as for delivery services. E-retailers are free to pass through the cost of this input to the end-consumers as they want: at a lower price than the actual cost they incurred, at cost or even by taking a positive margin on the delivery service. In a companion paper on the pricing strategies of e-retailers regarding shipping fees, Borsenberger et al. (2013) provide evidence that e-consumers will not react in the same manner whether shipping fees are charged explicitly or displayed in an "all-inclusive" form including both the price of the product and the price of the delivery services associated to the online purchase.

Developing a model grasping the value chain and the competitive interactions in the e-commerce sector is quite a challenge, and this chapter takes a first step in that direction. Specifically, we concentrate on delivery services.

While consumers can usually have their e-commerce purchases delivered to their home, an alternative mode of distribution has emerged over the last decades (initially for mail-order products) namely, delivery to a "relay point" (pick-up and drop-off location, for example in small stores like laundries, newspaper shops, gas stations). The principle is relatively simple: the parcel is shipped to a partner shop or business, which then hands it over to the end-customer. While home delivery is of course more convenient if it is carried out with success, customers who know that they are unlikely to be at home during normal delivery hours may prefer delivery to a relay point nearby (and with convenient opening hours). ${ }^{1}$ These two modes of delivery differ quite drastically in their cost structure. Specifically, the fixed part of total costs is significantly larger for the relay point mode of delivery, closest to a B2B logistic relationship (with the daily delivery of a preset number of points). The acquisition cost of a relay point (affiliation of a retailer, equipment) is fixed and the transportation cost of parcels to the relay points network is a predominantly fixed, function of the number of stops made during the route to supply the relays. The volume has a very low impact of the delivery cost of relay points. Only the retailer's

\footnotetext{
${ }^{*}$ Economist, European and National Regulation Division, Le Groupe La Poste.

${ }^{\dagger}$ Corresponding author: Toulouse School of Economics (IDEI, GREMAQ and IuF), helmuth.cremer@tse-fr.eu.

* Toulouse School of Economics (IDEI and GREMAQ-CNRS).

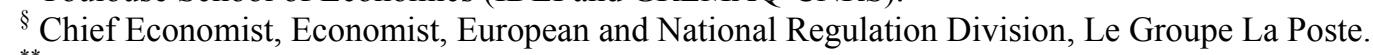

** Economist, European and National Regulation Division, Le Groupe La Poste.
} 
remuneration is variable (dependent on the number of parcels dropped off in its shop).

We study the pricing problem of a postal operator who sells delivery services to e-commerce firms that are perfectly competitive. This assumption, which implies that delivery rates are fully passed through to final consumers, is clearly highly restrictive and justified by the fact that we want to concentrate on the delivery segment. We start with two benchmark cases. In the first setting, there is a single operator who offers the two delivery technologies (Section 2). One can think of this as the incumbent (historic) postal operator. In Europe many of these operators continue to be at least in part publicly owned and often have a special status as Universal Service providers and face various public service obligations, so we assume that the single operator maximizes welfare rather than profits. Consequently, the pricing analysis here essentially reduces to a Ramsey problem. The second benchmark, in Section 3, is that where the incumbent postal operator offers only home delivery while an entrant delivers only to relays. We determine the Nash equilibrium of the (simultaneous) pricing game. Taste differences for the two delivery modes are represented by a Hotelling model in which consumers are distributed over an interval, where "location" zero corresponds to home delivery while one corresponds to delivery at a relay point. We study Ramsey prices for generic levels of the profit constraint, a specification that includes profit maximization as a special case.

Then we turn in Section 4 to a more complex duopoly setting in which the incumbent postal operator, who now offers both delivery services competes with a firm offering only delivery to relay points. This theoretical framework is close to the situation observed in many European countries, where the postal incumbent, the benchmark operator in the home delivery segment, has developed its own relay points network to compete with new players in the parcel delivery market segment who enter the market under the impulse of B2C e-commerce and propose delivery services in relay points, a network less costly to develop and satisfying needs of some customers. The incumbent postal operator continues to solve a Ramsey problem while the competitor maximizes profit. Once again, we determine the Nash equilibrium of the (simultaneous) pricing game. We now have three locations, 0,1 and $y$. We look at the scenario where $y$ represents the competitor's product while 0 and 1 continue to be the incumbent's home and relay point delivery. While we adopt the simplest possible model that is consistent with the main stylized features of the underlying problem, the analytical solutions are often quite complex. Their interpretation is interesting in itself, but to obtain sharper conclusion we resort to numerical simulations presented in Section 5. These are based on a specific model in which the cost structure and the distribution of tastes are set to achieve market shares that are consistent with a stylized representation of the currently observed market structure in many European countries. Section 6 concludes. Before proceeding we should point out that while our model (both the analytical part and the simulation) provides a good representation of the situation in many European countries, it does not provide a good description of the US postal market.

\section{MONOPOLY WITH TWO DELIVERY TECHNOLOGIES}

Consider a single e-commerce product $x$ which can be delivered either at home (index $h$ ) or in a relay (index $r$ ). The marginal cost of this product (excluding shipping costs) is constant and denoted by $k$; there is no fixed cost. Throughout this paper we assume that the e-commerce sector is perfectly competitive so that prices equal marginal costs (shipping included).

There is a single postal operator, indexed $P$, offering both modes of delivery. While parcel markets are typically not subject to direct regulation, we assume as noted above that the objective of the incumbent postal operator in these markets is not simply profit maximization. Specifically, we assume that operator $P$ maximizes welfare subject to some minimum profit requirement. This is effectively equivalent to assuming that it maximizes a weighted sum of consumer and producer surplus, with simple profit maximization as a special case. ${ }^{2}$ Consumers differ in their preferences over home versus relay delivery. To represent these taste differences we assume that consumers are distributed over a Hotelling line, with $z \in[0,1]$, according to distribution function $G(z)$ and density $g(z)$. Home delivery is the preferred option of the consumer at 0 , while delivery in a relay is the most preferred option of consumer at 1 . This specification is clearly a shortcut that relies on a highly stylized representation of reality. The "location" of a consumer in our model effectively captures a variety of factors including attachment to home delivery (which depends on the likelihood that a successful home delivery can occur) but also the proximity and accessibility of the closest relay point. We collapse all these dimensions of heterogeneity 
into a single one to keep the model tractable. It is important to keep this aspect in mind for the interpretation of our results.

Let $q_{0}$ denote the consumer price for the home delivered e-mail product set by the e-retailer, while $q_{1}$ represents the consumer price for the relay delivered product. Utility of consumer $z$ who buys $x$ units of the good is given by

$$
\begin{cases}u(x)-q_{0} x-t z^{2} & \text { if the good is delivered at home } \\ u(x)-q_{1} x-t(1-z)^{2} & \text { if the good is delivered in a relay }\end{cases}
$$

Define indirect utility (consumer surplus) as

$$
v(q)=\max _{x} u(x)-q x
$$

The solution to this problem also gives a consumer's demand function $x(q)$, which does not depend on location.,

The marginal consumer $\hat{z}\left(q_{0}, q_{1}\right)$ is defined by

$$
v\left(q_{0}\right)-t(\hat{z})^{2}=v\left(q_{1}\right)-t(1-\hat{z})^{2}
$$

In words this consumer is indifferent between home delivery (at price $q_{0}$ ) and relay delivery (at price $q_{1}$ ). All consumers to the left of $\hat{z}$ (with locations in $[0, \hat{z}[$ ) will opt for home delivery while those to the right (in $[\hat{z}, 1]$ ) will choose the relay mode. These two intervals then determine the market shares of the two delivery modes, which will be given by $G(\hat{z})$ for home and $1-G(\hat{z})$ for relay delivery respectively. Solving for $\hat{z}$ yields

$$
\hat{z}\left(q_{0}, q_{1}\right)=\frac{1}{2}+\frac{v\left(q_{0}\right)-v\left(q_{1}\right)}{2 t} .
$$

This expression is quite intuitive. When $q_{0}=q_{1}$ the marginal consumer is located at $1 / 2$. Note that (unless the distribution of consumers is uniform) this does not imply that the market shares of the two delivery modes are equal. We have

$$
\begin{aligned}
& \frac{\partial \hat{\mathrm{z}}}{\partial q_{0}}=-\frac{x_{0}}{2 t}, \\
& \frac{\partial \hat{\mathrm{z}}}{\partial q_{1}}=\frac{x_{1}}{2 t},
\end{aligned}
$$

where $x_{0}=x\left(q_{0}\right)$ and $x_{1}=x\left(q_{1}\right)$; similar short notation will be used throughout the paper as long as no confusion can arise.

Aggregate (market) demand for the two products is given by

$$
\begin{gathered}
X_{0}\left(q_{0}, q_{1}\right)=x\left(q_{0}\right) G\left[\hat{z}\left(q_{0}, q_{1}\right)\right], \\
X_{1}\left(q_{0}, q_{1}\right)=x\left(q_{1}\right)\left(1-G\left[\hat{z}\left(q_{0}, q_{1}\right)\right]\right) .
\end{gathered}
$$

The cost function of the delivery operator is expressed by 


$$
C=c_{h} X_{0}+c_{r} X_{1}+F_{h}+F_{r}+F
$$

where the subscripts $h$ and $r$ are used to identify the marginal and fixed costs associated with home and relay delivery respectively. The decomposition of fixed costs will not affect the first-order conditions (FOCs) for the Ramsey problem but it is useful to calibrate the model for the numerical simulations presented in Section 5.

\subsection{Ramsey solution}

Let $p_{0}$ and $p_{1}$ denote the respective linear shipping rates for home and relay delivery paid by the e-retailer to the delivery operator. Under perfect competition in the e-commerce sector, we have $q_{0}=k+p_{0}$ and $q_{1}=k+p_{1}$. Consequently the shipping costs incurred by the e-retailers are fully passed through to the final consumer. ${ }^{4}$

The Lagrangean expression associated with the Ramsey problem can be written as follows

$$
\begin{aligned}
& L_{2}=\int_{0}^{\hat{z}}\left[v\left(q_{0}\right)-t z^{2}\right] g(z) d z+\int_{\hat{z}}^{1}\left[v\left(q_{1}\right)-t(1-z)^{2}\right] g(z) d z \\
& +(1+\lambda)\left[p_{0} X_{0}+p_{1} X_{1}-c_{h} X_{0}-c_{r} X_{1}-F_{h}-F_{r}-F\right]-\lambda \bar{\pi},
\end{aligned}
$$

where $\bar{\pi}$ is the minimum profit requirement imposed on the postal operator.

The FOCs are given by

$$
\begin{aligned}
& \frac{\partial L_{2}}{\partial p_{0}}=-x_{0} G(\hat{z})+(1+\lambda)\left[X_{0}+\left(p_{0}-c_{h}\right) \frac{\partial X_{0}}{\partial q_{0}}+\left(p_{1}-c_{r}\right) \frac{\partial X_{1}}{\partial q_{0}}\right]=0 \\
& \frac{\partial L_{2}}{\partial p_{1}}=-X_{1}[1-G(\hat{z})]+(1+\lambda)\left[X_{1}+\left(p_{1}-c_{r}\right) \frac{\partial X_{1}}{\partial q_{1}}+\left(p_{0}-c_{h}\right) \frac{\partial X_{0}}{\partial q_{1}}\right]=0 .
\end{aligned}
$$

Rearranging and denoting the elasticity of aggregate demand by $\varepsilon$ yields the following Ramsey type expressions for the optimal prices:

$$
\begin{aligned}
& \frac{p_{0}-c_{h}}{q_{0}}=\frac{\lambda}{1+\lambda} \frac{1}{\left|\varepsilon_{0}\right|}-\frac{\left(p_{1}-c_{r}\right)}{q_{1}} \frac{\partial X_{1} / \partial q_{0}}{\partial X_{0} / \partial q_{0}} \\
& \frac{p_{1}-c_{r}}{q_{1}}=\frac{\lambda}{1+\lambda} \frac{1}{\left|\varepsilon_{1}\right|}-\frac{\left(p_{0}-c_{h}\right)}{q_{0}} \frac{\partial X_{0} / \partial q_{1}}{\partial X_{1} / \partial q_{1}} .
\end{aligned}
$$

These conditions are quite standard. The first terms are the usual inverse elasticity terms while the second terms arise because the demands for $X_{0}$ and $X_{1}$ depend on the price of the alternative delivery mode. They can be further simplified and rearranged by making use of the various expressions above (introducing displacement ratios or superelasticities) but this would add much for our purpose. One can easily verify that when $c_{h}=c_{r}$ and we get $q_{0}=q_{1}$ for any distribution of $z$ which satisfies $G(1 / 2)=1 / 2$. We now turn to the second reference case.

Let us now assume that there are two operators. One of them, labeled $P$ is the incumbent postal operator who only delivers at home. The competing operator (entrant), $E$, only delivers to relays. Its cost function has the same structure as (5), with a constant marginal cost of $c_{r}^{E}$ and a fixed cost $F^{E}$. We 
continue to use the same Hotelling specification with the two locations 0 for home delivery and 1 for relay delivery. We keep the same notation for prices as in the monopoly case: the subscript 0 or 1 represents the location. Most of the expressions derived in the previous section, including that for the marginal consumer, (2) and those for the aggregate demands, (3) and (4) remain valid.

We characterize the Nash equilibrium of this game where operator $P$ sets $q_{0}$, while operator $E$ (simultaneously) sets $q_{1}$. In equilibrium each operator's price must be the best-response to the other operator's strategy. To determine the best-response function we now study the pricing problem of each of the operators.

The objective function of operator $P$ is now to maximize the social welfare and is represented by the following Lagrangean expression

$$
\begin{aligned}
L_{D 2}= & \int_{0}^{\hat{z}}\left[v\left(q_{0}\right)-t z^{2}\right] g(z) d z+\int_{\hat{z}}^{1}\left[v\left(q_{1}\right)-t(1-z)^{2}\right] g(z) d z \\
& +(1+\lambda)\left[p_{0} X_{0}-c_{h} X_{0}-F_{h}-F^{P}\right]-\lambda \bar{\pi}+\pi_{D 2}^{E},
\end{aligned}
$$

where

$$
\pi_{D 2}^{E}=\left(p_{1}-c_{r}^{E}\right) X_{1}-F^{E}
$$

is operator $E$ 's profit. This expression differs from its counterpart in the monopoly case, (6), in that profits associated with the relay technology, $\pi_{D 2}^{E}$, are not multiplied by $(1+\lambda)$; this is normal because they now go to the other operator and no longer contribute to the budget balancing of operator $P$. Furthermore the marginal cost of relay delivery is now $c_{r}^{E}$. Differentiating (9) with respect to $q_{0}$ and rearranging yields

$$
\frac{p_{0}-c_{h}}{q_{0}}=\frac{\lambda}{1+\lambda} \frac{1}{\left|\varepsilon_{0}\right|}-\frac{\left(p_{1}-c_{r}^{E}\right)}{(1+\lambda) q_{0}} \frac{\partial X_{1} / \partial q_{0}}{\partial X_{0} / \partial q_{0}} .
$$

Operator $E$ on the other hand maximizes its profit. Differentiating (10) with respect to $q_{1}$ and rearranging yields

$$
\frac{p_{1}-c_{r}^{E}}{q_{1}}=\frac{1}{\left|\varepsilon_{1}\right|}
$$

Expression (12) is well known; it is the typical pricing expression for a profit-maximizing firm in a differentiated oligopoly. Expression (11) is also rather standard but deserves more attention. The first term on the right-hand side is the traditional Ramsey (inverse elasticity) expression. Since (12) implies $p_{1}>c_{r}^{E}$, and given that we have of course $\partial X_{0} / \partial q_{0}<0$ and $\partial X_{1} / \partial q_{0}>0$, the second term (including the minus sign) is positive. This means that even when $\lambda=0$ (markups are not justified by the need to finance fixed costs), operator $P$ would set its price above marginal costs. This is done to mitigate the distortion created by the other operator's pricing behavior. Increasing the price will of course reduce individual demand levels (which is bad for welfare) but it will also mitigate the distortion in the marginal consumer (since the price of $E$ is too high, too many consumers opt for home delivery). ${ }^{5}$ All this means in turn that when fixed costs are not too large, we might indeed have a solution where the break-even constraint is not binding. This occurs when the distortion-mitigating markup is large enough to cover the fixed costs. An illustration of the range of minimum profit requirement $\bar{\pi}$ under which this regime occurs is provided in the numerical examples presented in Section 5.

Further observe that when the break-even constraint binds, the pricing problem is even simpler than in the monopoly case. Since operator $P$ has a single instrument we can obtain $q_{0}$ (as a function of the 
other operator's price) simply by solving the break-even constraint. Expression (11) then effectively only determines the level of $\lambda$.

We now turn to the case where both operators offer relay delivery.

We continue to assume that there are two operators. However, operator $P$ now uses both technologies. The competing operator (entrant), E, only delivers to relays. We continue to use the same Hotelling specification allowing, however, for three locations $0, y$ (with $0<y<1$ ) and 1 . We keep the same notation for prices as in the previous sections: the subscript represents the location (characteristic of horizontal differentiation): $0, y$ or 1 . We concentrate on a setting where product 0 represents home delivery, 1 is the operator $P$ (the incumbent)'s relay delivery, while operator $E$ supplies product $y$ (relay delivery). When $y$ is different from 1 , the two relay networks are not perfect substitutes. This may be due to reputational issues but also to the location and number of the networks' respective relay points. In any event, we model this as equivalent to an exogenously determined service offering "in between" what we might call "pure" home delivery" and "pure" relay. ${ }^{6}$

\section{Preferences and demand}

The utility of consumer $z$, who buys $x$ units of the good, is given by

$$
\begin{cases}u(x)-q_{0} x-t z^{2} & \text { if the good is delivered according to characteristic } 0, \\ u(x)-q_{y} x-t(y-z)^{2} & \text { if the good is delivered according to characteristic } y, \\ u(x)-q_{1} x-t(1-z)^{2} & \text { if the good is delivered according to characteristic } 1 .\end{cases}
$$

The indirect utility (surplus) continues to be defined by (1). The new feature is that we now (potentially) have two marginal consumers: $\hat{Z}_{0 y}\left(q_{0}, q_{y}\right)$ and $\hat{Z}_{y 1}\left(q_{y}, q_{1}\right)$. The first one is indifferent between 0 and $y$. Consequently, $\hat{Z}_{0 y}\left(q_{h}, q_{y}\right)$ is the solution to

which yields

$$
v\left(q_{0}\right)-t z^{2}=v\left(q_{y}\right)-t(y-z)^{2}
$$

$$
\hat{Z}_{0 y}\left(q_{0}, q_{y}\right)=\frac{y}{2}+\frac{v\left(q_{0}\right)-v\left(q_{y}\right)}{2 t} .
$$

The second one, $\hat{z}_{y 1}\left(q_{y}, q_{1}\right)$, is indifferent between $y$ and 1 and is the solution to

$$
v\left(q_{y}\right)-t(z-y)^{2}=v\left(q_{1}\right)-t(1-z)^{2} .
$$

Solving this expression we obtain

$$
\hat{z}_{y 1}\left(q_{y}, q_{1}\right)=\frac{1+y}{2}+\frac{v\left(q_{y}\right)-v\left(q_{1}\right)}{2 t(1-y)}
$$

Aggregate demands for the different products are given by

$$
\begin{aligned}
& X_{0}\left(q_{0}, q_{y}\right)=x\left(q_{0}\right) G\left[\hat{z}_{0 y}\left(q_{0}, q_{y}\right)\right], \\
& X_{y}\left(q_{0}, q_{y}, q_{1}\right)=x\left(q_{y}\right)\left(G\left[\hat{z}_{y 1}\left(q_{y}, q_{1}\right)\right]-G\left[\hat{z}_{0 y}\left(q_{0}, q_{y}\right)\right]\right), \\
& X_{1}\left(q_{y}, q_{1}\right)=x\left(q_{1}\right)\left(1-G\left[\hat{z}_{y 1}\left(q_{y}, q_{1}\right)\right]\right) .
\end{aligned}
$$


We continue to assume that the operator $P$ solves a Ramsey problem (which has profit maximization as a special case), while operator $E$ maximizes profits. We characterize the Nash equilibrium of this game assuming that all delivery rates are set simultaneously. The cost function of the operator $E$ has the same structure as (5), with a constant marginal cost of $c_{r}^{E}$ and a fixed cost $F^{E}$. The relevant cost parameters for operator $P$ are now denoted $c_{h}, C_{r}^{P}, F_{h}, F_{r}^{P}$ and $F^{P}$ (adding the superscript $P$ where necessary). We now provide a detailed study of the most relevant configuration.

\section{Equilibrium}

The configuration we study is shown in the following figure

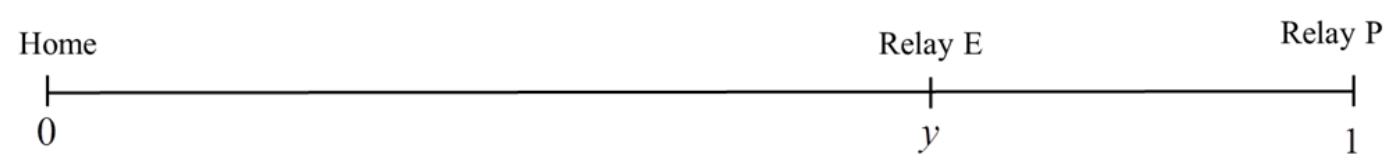

Figure 1: Configuration of operators

The Lagrangean expression associated with the Ramsey problem of operator $P$ (who chooses $p_{0}$ and $p_{1}$, given $\left.p_{y}\right)$ can be written as follows

$$
\begin{aligned}
L_{C 1} & =\int_{0}^{\hat{z}_{0 y}}\left[v\left(q_{0}\right)-t z^{2}\right] g(z) d z+\int_{\hat{z}_{y 1}}^{1}\left[v\left(q_{1}\right)-t(1-z)^{2}\right] g(z) d z \\
& +\int_{\hat{z}_{0 y}}^{\hat{z}_{y 1}}\left[v\left(q_{y}\right)-t(y-z)^{2}\right] g(z) d z \\
& +(1+\lambda)\left[p_{0} X_{0}+p_{1} X_{1}-c_{h} X_{0}-c_{r}^{P} X_{1}-F_{h}-F_{r}^{P}-F^{P}\right]-\lambda \bar{\pi}+\pi_{C 1}^{E},
\end{aligned}
$$

where the arguments of $\hat{Z}_{0 y}\left(q_{0}, q_{y}\right), \hat{Z}_{y 1}\left(q_{y}, q_{1}\right), X_{0}\left(q_{0}, q_{y}\right)$ and $X_{1}\left(q_{y}, q_{1}\right)$ have been omitted for simplicity (but one has to keep them in mind) and where

$$
\pi_{C 1}^{E}=\left(p_{y}-c_{r}^{E}\right) X_{y}\left(q_{0}, q_{y}, q_{1}\right)-F^{E}
$$

is operator's $E$ profit, and where consumer prices (of the e-commerce product $x$ ) equal marginal costs, including shipping fees, so that $q_{0}=k+p_{0}, q_{1}=k+p_{1}$ and $q_{y}=k+p_{y}$. This is exactly like in the monopoly case and follows from our assumption of perfect competition and constant marginal costs in the e-commerce sector. Observe that operator $P$ maximizes welfare (total surplus) which includes operator $E$ 's profit. However, $E$ 's profit receives a lower weight than that of operator $P ; 1$ as opposed to $(1+\lambda)$. This is because an increase in operator $P$ 's profit relaxes the break-even constraint.

To determine operator $P$ 's best-reply function, we maximize $L_{C 1}$ with respect to $p_{0}$ and $p_{1}$, given $p_{y}$ (set by operator $E$ ). Interestingly the two market segments are now completely independent. They both depend on $E$ 's price, but this variable is considered as a constant at this point; recall that we determine a Nash equilibrium where operator $P$ 's prices must be the best reply to those of operator $E$ (and vice versa). Consequently we get a Ramsey solution with independent demands. ${ }^{7}$ However, the competitor's profit depends on P's prices, which bring in an extra term on top of the inverse elasticity one. Optimal prices are defined by ${ }^{8}$

$$
\frac{p_{0}-c_{h}}{q_{0}}=\frac{\lambda}{1+\lambda} \frac{1}{\left|\varepsilon_{0}\right|}-\frac{\left(p_{y}-c_{r}^{E}\right)}{(1+\lambda) q_{0}} \frac{\partial X_{y} / \partial q_{0}}{\partial X_{0} / \partial q_{0}},
$$




$$
\frac{p_{1}-c_{r}^{P}}{q_{1}}=\frac{\lambda}{1+\lambda} \frac{1}{\left|\varepsilon_{1}\right|}-\frac{\left(p_{y}-c_{r}^{E}\right)}{(1+\lambda) q_{1}} \frac{\partial X_{y} / \partial q_{1}}{\partial X_{1} / \partial q_{1}}
$$

Note that the second term on the right-hand side vanishes when $P$ maximizes profit (which formally translates into $\lambda \rightarrow \infty$ ).

These expressions (along with the break even constraint) define operator $P$ 's prices as functions of $p_{y}$. This variable comes in through the price elasticities and the last term. The comparative statics appear to be complicated analytically. The relevant question is the sign of the derivative, which tells us how operator $P^{\prime}$ s price is affected by an increase in operator $E$ 's price. When the best response function is increasing the modes of delivery are said to be strategic complements; otherwise, they are strategic substitutes. This property is important to understand intuitively how a change in the parameters affects the market equilibrium. We shall examine this question in the numerical section.

Operator $E$ maximizes its profit given by (16) with respect to $p_{y}$ (given the other two prices). This yields the usual pricing expression (for differentiated Bertrand competition), which can be written as

$$
\frac{p_{y}-c_{r}^{E}}{q_{y}}=\frac{1}{\left|\varepsilon_{y}\right|} \text {. }
$$

Equations (17), (18) and (19) along with the break even constraint determine the Nash equilibrium prices (and the equilibrium level of $\lambda$ ). Like in Section 3 and for the same reasons as discussed there, we can again have two possible regimes: one where $\lambda=0$ (break-even constraint not binding) which occurs for "low" levels of fixed costs or of profit requirement $\bar{\pi}$, and one where $\lambda>0$. In the latter case the fixed costs are too large to be covered by the markups implied by duopoly competition. Recall that operator $P$ tends to set positive markups to mitigate distortions in consumer allocation which in turn arise because the profit-maximizing operator $E$ always sets a price above marginal costs.

\section{$5 \quad$ NUMERICAL SIMULATIONS}

We now provide numerical simulations of our model when the cost structure and the distribution of tastes are set to achieve market shares consistent with a stylized representation of the currently observed market structure. These simulations allow us to go beyond the formulas developed in the preceding sections to shed light on the equilibrium levels of variables (such as prices, volumes and market shares, profits, consumer surplus and welfare). This in turn informs us about the relative sizes of the effects mentioned above. For instance, we investigate what levels of fixed costs can be covered by the mark-up over marginal costs posted by a pure welfare maximizing postal operator. Also, we study how results are affected when the distribution of consumers tastes changes (for instance in favor of relays as time passes).

Assume that the distribution of consumers follows a Pareto distribution truncated over [0,1], with parameter $\alpha$, and that the utility function $u(x)$ is quadratic, so that individual demand functions $x(q)$ are linear. We start with the monopoly situation.

With these assumptions, in the monopoly model, the market share of home delivery depends only on the value of $\alpha$ when $q_{0}=q_{1}$. Assuming that this market share is $90 \%$, we obtain a value of $\alpha$ of 5.1. We will also consider a lower value of $\alpha(\alpha=3)$ which, as can be seen from Figure 2, corresponds to a less skewed distribution of consumer preferences, with fewer consumers located close to home delivery and more people located close to the relay delivery at location one. When $\alpha=3,80 \%$ of consumers favor home to relay delivery when both are priced equally. 


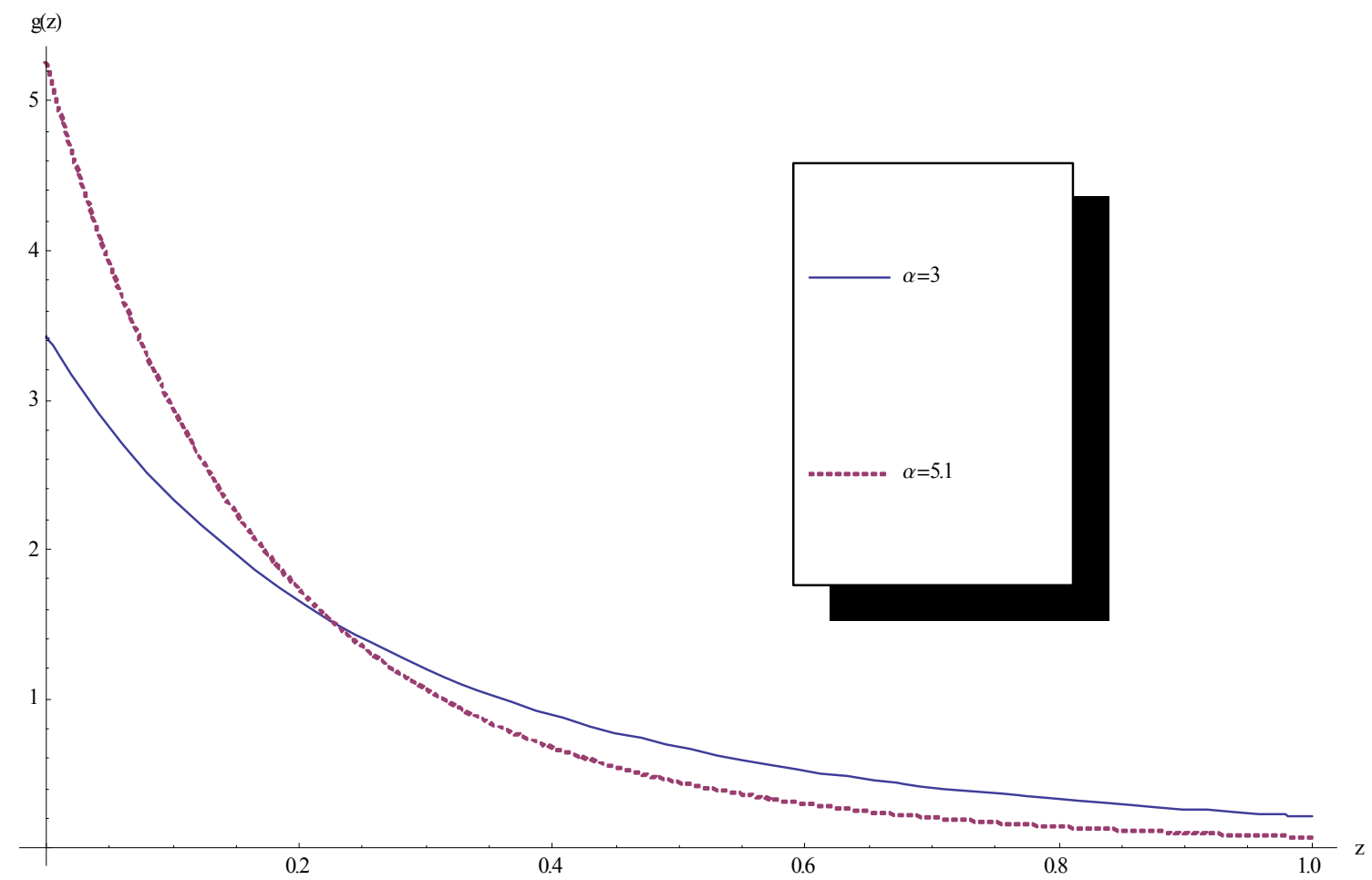

Figure 2. Consumers density function with $\alpha=5.1$ and $\alpha=3$

To calibrate the value of the transportation cost $t$ and of the two parameters of the utility function, we assume $\alpha=5.1, k=10$ and that $p_{0}=p_{1}=4$, so that $q_{0}=q_{1}=14$. We further make use of the following three assumptions: (1) the total volume delivered at these prices, $X_{0}\left(q_{0}, q_{1}\right)+X_{1}\left(q_{0}, q_{1}\right)$ equals 250 (million items); (2) the market share of home delivery is $58 \%$ when home delivery is $40 \%$ more expensive than delivery in a relay, while it is $95 \%$ when delivery in a relay is $18 \%$ more expensive. As for costs, we assume that the marginal cost of home delivery is, at $c_{h}=1.5,50 \%$ more expensive than delivery in a relay $\left(c_{r}=1\right)$. There are no fixed costs $\left(F_{h}+F_{r}+F=0\right)$. Instead we vary the minimum profit level required of the postal operator, $\bar{\pi}$. This allows us to compare the welfare levels across simulations varying in $\bar{\pi}$.

\subsection{Monopoly}

Table 1 presents the monopoly solution as a function of the minimum profit level requirement for the postal operator, $\bar{\pi}$.

The first column of Table 1 corresponds to the case where $\bar{\pi}=0$, so that the welfare maximizing monopolist sets prices at marginal cost $\left(p_{0}=c_{h}, p_{1}=c_{r}\right)$. Even though the delivery price at home is $50 \%$ more expensive than in relays, home delivery retains more than three fourths of the market. As the profit requirement $\bar{\pi}$ increases, both prices increase. Volumes, consumer surplus and welfare then decrease, while the market share of the home delivery decreases very slowly. The last column of Table 1 reports the profit-maximizing allocation (when $\lambda=\infty$ ), corresponding to a profit of 657 .

\begin{tabular}{|c|c|c|c|c|c|}
\hline $\bar{\pi}$ & 0 & 100 & 300 & 650 & 657 \\
\hline$p_{0}$ & 1.50 & 1.74 & 2.29 & 4.22 & 4.56 \\
\hline$p_{1}$ & 1.00 & 1.22 & 1.73 & 3.36 & 3.63 \\
\hline$\hat{z}$ & 0.328 & 0.329 & 0.330 & 0.325 & 0.328 \\
\hline Consumer surplus & 1282 & 1179 & 959 & 373 & 298 \\
\hline
\end{tabular}




\begin{tabular}{|c|c|c|c|c|c|}
\hline Welfare & 1282 & 1279 & 1259 & 1023 & 955 \\
\hline$X_{0}$ & 342 & 329 & 296 & 183 & 164 \\
\hline$X_{1}$ & 100 & 96 & 88 & 64 & 59 \\
\hline$X_{0}+X_{1}$ & 442 & 425 & 384 & 247 & 223 \\
\hline$X_{0} /\left(X_{0}+X_{1}\right)$ & $77.41 \%$ & $77.39 \%$ & $77.20 \%$ & $74.17 \%$ & $73.65 \%$ \\
\hline$\lambda$ & 0 & 0.043 & 0.177 & 4.038 & $\infty$ \\
\hline
\end{tabular}

Table 1: Ramsey allocation as a function of fixed costs under monopoly with $\alpha=5.1$

We now check how these results are affected when consumers tastes change in favor of relay delivery (i.e., when $\alpha=3$ ). In order to isolate the impact of the distribution function on the results, we keep the other parameters of the calibration ( $t$ and the two parameters in the utility function) constant. ${ }^{10}$ The results are reported in Table 2 .

Once again, both prices increase with $\bar{\pi}$, while the market share of home delivery decreases very slowly. We then compare the two tables, starting with the first numerical column. With identical prices in the two tables, the home delivery volumes decrease less (from 342 to 284 million items) than the increase in the relay delivery volumes (from 100 to 163), so that total volumes increase (from 442 to 447 ) when the distribution of consumers tastes changes in favor of relay delivery. The shift of volumes from home to relay delivery is due to the fact that the marginal consumer is the same in both cases, with a smaller fraction of agents located to the left of $\hat{z}$ when $\alpha$ decreases from 5.1 to 3 . Since the consumer price with home delivery is cheaper than that with relay delivery, consumers who shift from home delivery to relay delivery increase their demand and total volume increases. Consequently, consumer surplus and total welfare are higher when consumers have an intrinsic preference for the less costly delivery technology.

\begin{tabular}{|c|c|c|c|c|c|}
\hline $\bar{\pi}$ & 0 & 100 & 300 & 650 & 670 \\
\hline$p_{0}$ & 1.50 & 1.74 & 2.29 & 4.07 & 4.61 \\
\hline$p_{1}$ & 1.00 & 1.22 & 1.73 & 3.27 & 3.75 \\
\hline$\hat{z}$ & 0.328 & 0.329 & 0.331 & 0.334 & 0.345 \\
\hline Consumer surplus & 1297 & 1195 & 975 & 421 & 295 \\
\hline Welfare & 1297 & 1295 & 1275 & 1071 & 965 \\
\hline$X_{0}$ & 284 & 273 & 247 & 162 & 138 \\
\hline$X_{1}$ & 163 & 156 & 143 & 103 & 88 \\
\hline$X_{0}+X_{1}$ & 447 & 430 & 390 & 265 & 226 \\
\hline$X_{0} /\left(X_{0}+X_{1}\right)$ & $63.63 \%$ & $63.61 \%$ & $63.41 \%$ & $61.11 \%$ & $61.15 \%$ \\
\hline$\lambda$ & 0 & 0.042 & 0.171 & 2.331 & $\infty$ \\
\hline
\end{tabular}

Table 2: Ramsey allocation as a function of fixed costs under monopoly when $\alpha=3$

Comparing the next columns, shows that prices remain similar for values of $\bar{\pi}$ lower than 650 . The markup over marginal cost is larger for home delivery than for relay delivery. Transferring consumers from home to relay delivery then decreases the aggregate contribution of the two delivery modes to profit, for given prices and aggregate volume. At the same time, the larger total volume, at given prices, makes it easier to generate profit. The two opposite effects nearly cancel each other when $\bar{\pi}$ is lower than 650 , so that prices are similar. Prices become noticeably smaller for $\bar{\pi}=650$ when consumers are more interested in the relay option, with the maximum feasible amount of $\bar{\pi}$ is larger in Table 2 than in Table 1. 


\subsection{Duopoly with single technology operators}

Consider a duopoly with single technology operators, where the postal operator, delivering at home, faces the competition of an entrant operating a relay located at one. To ease comparison, we keep the calibration of the parameters. Table 3 reports the Ramsey allocations, when $\alpha=5.1$, as a function of the profit requirement $\bar{\pi}$ assigned to the postal operator.

\begin{tabular}{|c|c|c|c|c|}
\hline $\bar{\pi}$ & $\leq 126$ & 150 & 200 & 245 \\
\hline$p_{0}$ & 1.86 & 1.94 & 2.15 & 2.59 \\
\hline$p_{1}$ & 1.63 & 1.63 & 1.63 & 1.60 \\
\hline$\hat{z}$ & 0.429 & 0.404 & 0.341 & 0.207 \\
\hline Consumer surplus & 1108 & 1079 & 1012 & 896 \\
\hline Welfare & 1271 & 1270 & 1266 & 1235 \\
\hline$X_{0}$ & 352 & 341 & 309 & 225 \\
\hline$X_{1}$ & 58 & 65 & 85 & 155 \\
\hline$X_{0}+X_{1}$ & 410 & 405 & 394 & 380 \\
\hline$X_{0} /\left(X_{0}+X_{1}\right)$ & $85.91 \%$ & $84.06 \%$ & $78.42 \%$ & $59.08 \%$ \\
\hline$\Pi_{D 2}^{E}$ & 37 & 41 & 54 & 94 \\
\hline$\lambda$ & 0 & 0.032 & 0.162 & $\infty$ \\
\hline
\end{tabular}

Table 3: Ramsey allocation with single technology operators and $\alpha=5.1$

In the first numerical column, we set exogenously $\lambda$ at zero. The entrant's price is much larger than marginal cost, and the welfare-maximizing operator reacts by increasing its price to mitigate distortions in the allocation of consumers across delivery methods (see Section 3). Since the postal operator's price exceeds marginal cost $\left(p_{0}>c_{h}\right)$, the operator makes a profit of 126 million euros. As long as the profit requirement $\bar{\pi}$ is lower than this amount, this allocation remains the Ramsey optimum. Table 3 presents the solution when $\bar{\pi}$ is increased above this level. ${ }^{11}$ To achieve higher profits, the operator increases its price $p_{0}$. The entrant first raises its price, and then decreases it once a threshold is attained, since its reaction function is inversely $\mathrm{U}$-shaped in $p_{0}$. The entrant captures an increasing fraction of a lower total volume, with the identity of the marginal consumer moving to the left. The entrant's profit increases as the profit-constraint of the postal operator becomes more binding. Comparing the profit-maximizing monopoly with both technologies to the duopoly with single technology operators, we obtain the classical results: consumers are better off when competition is introduced (they face lower prices and demand increases). Overall, despite a lower producers' surplus, and despite $P$ maximizing welfare rather than profit, while $E$ is interested only its profit, welfare increases. ${ }^{12}$

Table 4 reports the results obtained when consumers tastes are shifted in favor of relay delivery ( $\alpha=5.1$ instead of $\alpha=3$ ).

\begin{tabular}{|c|c|c|c|}
\hline $\bar{\pi}$ & $\leq 161$ & 200 & 237 \\
\hline$p_{0}$ & 2.04 & 2.24 & 2.69 \\
\hline$p_{1}$ & 1.82 & 1.84 & 1.86 \\
\hline$\hat{z}$ & 0.433 & 0.380 & 0.261 \\
\hline Consumer surplus & 1032 & 973 & 865 \\
\hline Welfare & 1276 & 1274 & 1252 \\
\hline$X_{0}$ & 297 & 268 & 198 \\
\hline$X_{1}$ & 101 & 120 & 174 \\
\hline$X_{0}+X_{1}$ & 398 & 388 & 372 \\
\hline
\end{tabular}




\begin{tabular}{|c|c|c|c|}
\hline$X_{0} /\left(X_{0}+X_{1}\right)$ & $74.72 \%$ & $69.20 \%$ & $53.18 \%$ \\
\hline$\Pi_{D 2}^{E}$ & 83 & 101 & 150 \\
\hline$\lambda$ & 0 & 0.143 & $\infty$ \\
\hline
\end{tabular}

Table 4: Ramsey allocation with single technology operators and $\alpha=3$

Focusing on Table 4, we can reproduce all the comments made on Table 3, with one caveat: the profit-maximizing price of the entrant increases monotonically with $\bar{\pi}$, because the postal operator's price is low enough that the entrant is on the upward sloping part of its reaction function.

We now compare the first numerical columns of Tables 3 and 4. The change in consumers tastes benefits the entrant by increasing its volume for any given configuration of prices. When increasing its price, the entrant faces a trade-off between the larger revenue on its customer base and the loss of customers. Even though there are more switching customers when tastes are changed (as shown on Figure 2 when comparing the density of customers at $\hat{z}$ ), the effect linked to the larger customer base is more important and results in a larger profit-maximizing price. The postal operator reacts by pushing its price above the level attained in Table 3. Consequently, consumer surplus decreases. However, total welfare is slightly higher because the entrant more than doubles its profit; the postal operator's profit also increases as well, albeit by a smaller amount. Home delivery loses volumes to the benefit of relay delivery. We obtain similar results when the postal operator's profit requirement $\bar{\pi}$ is increased above 162 million euros. Interestingly, the maximum level of the postal operator's profit is lower in Table 4 than in Table 3.

\subsection{Duopoly with competition on relay delivery}

We now move to the duopoly where the postal operator offers both home delivery (at zero) and relay delivery (at one). Table 5 reports results when the entrant located at $y=0.75$ maximizes its profit while the postal operator posts Ramsey prices for both home and relay delivery with $\alpha=5.1$.

The first numerical column reports result when $\lambda=0$. Since the entrant's price is $37 \%$ larger than its marginal cost $\left(p_{y}>c_{r}^{E}\right)$, the postal operator also posts prices above marginal costs in order to mitigate the distortion in the allocation of consumers across delivery modes. The postal operator makes a 83 million $€$ profit (to be compared with 71 million $€$ for the entrant). Hence, as long as the profit requirement $\bar{\pi}$ is lower than 83 million $€$, this allocation remains the second-best optimal one with a profit-maximizing entrant.

\begin{tabular}{|c|c|c|}
\hline $\bar{\pi}$ & $\leq 83$ & 76 \\
\hline$p_{0}$ & 1.86 & 1.89 \\
\hline$p_{y}$ & 1.37 & 1.31 \\
\hline$p_{1}$ & 1.37 & 1.14 \\
\hline$\hat{z}_{0 y}$ & 0.16 & 0.12 \\
\hline$\hat{z}_{y 1}$ & 0.868 & 0.647 \\
\hline Consumer surplus & 1166 & 1176 \\
\hline Welfare & 1321 & 1320 \\
\hline$X_{0}$ & 226 & 185 \\
\hline$X_{y}$ & 192 & 222 \\
\hline$X_{1}$ & 6 & 23 \\
\hline$X_{0} /\left(X_{0}+X_{y}+X_{1}\right)$ & $53.32 \%$ & $42.98 \%$ \\
\hline$X_{1} /\left(X_{y}+X_{1}\right)$ & $2.80 \%$ & $9.54 \%$ \\
\hline$\Pi_{C 1}^{E}$ & 72 & 68 \\
\hline
\end{tabular}




\section{\begin{tabular}{|c|c|c|}
\hline$\lambda$ & 0 & $\infty$ \\
\hline
\end{tabular}}

Table 5: Ramsey allocation with duopoly, $y=0.75$ and $\alpha=5.1$

The second numerical column of Table 5 reports the allocation where the postal operator maximizes profit (i.e., where we exogenously set $\lambda=\infty$ in the Ramsey problem). Compared with welfare-maximization (first column), the postal operator chooses to increase slightly $p_{0}$ (to increase its mark-up, at the expense of volume) and to decrease substantially $p_{1}$ (to increase its volume at the expense of its mark-up). Faced with a significantly lower relay price by the postal operator, the entrant decreases its price $p_{y}$ compared to the first column. Profit-maximization by all then results in two striking observations. First, the marginal consumer $\hat{z}_{y 1}$ is located to the left of the entrant's position $y$ : the postal firm is so aggressive when setting its relay price $p_{1}$ that it captures all demand to the right of $0.647<y=0.75$. Second, the profit level of both operators is lower when they both maximize profit than when the postal operator maximizes welfare. This result (due to the strategic interactions between firms) may seem counterintuitive at first sight but is reminiscent of many such results found in the mixed oligopoly literature (which studies imperfect competition between firms differing in their objective, see for instance Matsumara 1998 and White 2002). Welfare is higher when the postal operator maximizes welfare with $\lambda=0$, but consumer surplus is higher when both firms maximize profits.

Table 6 reports the results for $\alpha=3$. We obtain results qualitatively similar to those in Table 5, both when $\lambda=0$ and when $\lambda=\infty$. Comparing with Table 5 , all prices are higher and volumes are higher for relays and lower for home delivery. Profit is larger for the entrant but smaller for the postal operator when the distribution of preferences moves in favor of relay delivery.

\begin{tabular}{|c|c|c|}
\hline $\bar{\pi}$ & $\leq 76$ & 64 \\
\hline$p_{0}$ & 1.92 & 1.89 \\
\hline$p_{y}$ & 1.42 & 1.32 \\
\hline$p_{1}$ & 1.41 & 1.17 \\
\hline$\hat{z}_{0 y}$ & 0.16 & 0.13 \\
\hline$\hat{z}_{y 1}$ & 0.87 & 0.66 \\
\hline Consumer surplus & 1169 & 1205 \\
\hline Welfare & 1347 & 1347 \\
\hline$X_{0}$ & 168 & 144 \\
\hline$X_{y}$ & 243 & 240 \\
\hline$X_{1}$ & 15 & 50 \\
\hline$X_{0} /\left(X_{0}+X_{y}+X_{1}\right)$ & $39.50 \%$ & $33.29 \%$ \\
\hline$X_{1} /\left(X_{y}+X_{1}\right)$ & $5.65 \%$ & $17.18 \%$ \\
\hline$\Pi_{C 1}^{E}$ & 102 & 78 \\
\hline$\lambda$ & 0 & $\infty$ \\
\hline
\end{tabular}

Table 6: Ramsey allocation with duopoly, $y=0.75$ and $\alpha=3$

E-commerce is a form of retailing on a growing trend, which differs from traditional brick-and-mortar retail by several aspects. In particular, its value chain involves the intervention of a delivery operator, who ensures the physical delivery of the product bought online to its final consumer. Between the three major actors of e-commerce, the e-retailer, the e-consumer and the delivery operator, there are clear and 
separate business relationships based on the principle of freedom to contract: on the one hand, the business relationship between the online shopping site and the service provider that delivers the parcels; on the other hand, the business relationship between the online shopping site and the final consumer.

In this paper, we focus on the relationship between e-retailers and delivery operators and study the pricing problem of a postal operator selling delivery services to perfectly competitive e-retailers. What are the optimal shipping prices set by the delivery operator, knowing that, in this special case, the delivery costs incurred by the e-retailer are fully passed through to the final consumers via shipping fees? We have considered several benchmark cases according to the number of delivery operators in the market (monopoly versus duopoly cases) and the delivery technologies offered (delivery at home or in a relay point). We have determined the Nash equilibrium of the pricing game under the assumption that consumers have heterogeneous preferences for the delivery mode of their online purchases (represented by a Hotelling model). From the analytical solutions and numerical simulations of the problem, we derived some "classical results". For instance, we obtain the usual inverse elasticity pricing formulas for profit maximizing operators. In addition, we show that introducing competition into a market with a single, profit-maximizing operator increases the consumers' surplus and the welfare.

We also obtain a number of interesting but less obvious results. ${ }^{13}$ In a monopoly setting with both delivery modes, when there are no fixed costs, the welfare-maximizing strategy of the delivery operator is to set the price of each delivery service at its marginal cost. As fixed costs are gradually increased, the optimal prices diverge from marginal costs and the share of demand for home delivery decreases slightly since the relay option is cheaper (the gap between the optimal prices of both options is increasing with fixed costs). When consumers' preferences for delivery in relay are stronger (without making it the dominant mode), the demand for delivery at home decreases as expected to the benefit of delivery in relay, but the total demand addressed to the delivery operator increases. This is because the consumer price of the good delivered in a relay is lower than when it is delivered at home; consequently, consumers who shift from home delivery to relay delivery increase their demand.

In a duopoly setting with a welfare-maximizing operator, who competes with a profit-maximizing entrant, the entrant sets a price for its service (relay delivery) above its marginal cost. This distorts the allocation of consumers across delivery modes compared to the social optimum (more consumers choose the alternative options to entrant's relay technology). In order to mitigate this effect, the welfare-maximizing incumbent increases its own prices (from home delivery and relay delivery when offering both technologies) above their marginal cost (but to a smaller degree than a profit-maximizing incumbent). Like in the monopoly setting, as consumers' preferences for the relay technology become stronger, the entrant can increase its price further without losing volumes and thus makes higher profits. To mitigate the distortionary effect on preferences allocation, the welfare-maximizing incumbent once again also increases its prices. Globally, the demand for delivery service decreases due to increase in prices and the share of delivery at home decreases.

\section{References}

[1] Borsenberger C., Joram D., Lécou S. and Ligouzat E., (2013), The pricing strategies of e-retailers regarding shipping fees, presented at the 21th Conference on Postal and Delivery Economics.

[2] Brousseau, E., (2003), E-commerce in France: did early adoption prevent its development?, The Information Society, 19(1), 45-57.

[3] Brynjolfsson E. and Smith M.D., (2000), Frictionless Commerce? A Comparison of Internet and Conventional Retailers, Management Science, 46(4), 563-585.

[4] Cabral L. and Hortaçsu A., (2010), The Dynamics of Seller Reputation: Evidence from eBay, Journal of Industrial Economics, 58(1), 54-78.

[] Clay K., Krishnan R. and Wolff E., (2002), Retail Strategies on the Web: Price and Non-price Competition in the Online Book Industry, Journal of Industrial Economics, 50(3), 351-367.

[5] Cremer, H, M. Marchand and J.-F. Thisse, (1991), Mixed oligopoly with differentiated products, International Journal of Industrial Organization, 9, 43-53.

[6] Feldstein M.S., (1972), The pricing of public intermediate goods, Journal of Public Economics, 1, 45-72.

[7] FEVAD, (2013), Les chiffres clés de la vente à distance, édition 2012.

[8] Javalgi, R. and Ramsey, R., (2001), Strategic issues of e-commerce as an alternative global 
distributions system, International Marketing Review, 18(4), 376-391.

[9] Matsumura T, (1998), Partial privatization in mixed duopoly, Journal of Public Economics, 70, 473-483.

[10] Spencer B.J. and Brander J.A., (1983), Second best pricing of publicly produced inputs, Journal of Public Economics, 20, 113-119.

[11] White M, (2002), Political manipulation of a public firm's objective function, Journal of Economic Behavior \& Organization, 49, 487-499.

\footnotetext{
${ }^{1}$ While relay delivery bears some resemblance with store delivery, it is different in that the number of replay points is quite large (in France Kiala a relay operator competing with La Poste has some 4,500 delivery points, while Pickup service, the relay network operated by La Poste has 5,800 points; no chain store has nearly as many outlets). Consequently, customers typically can find a relay point quite close to their home. Relay delivery is therefore a much closer substitute to home delivery than traditional store delivery.

${ }^{2}$ This case is obtained when the profit requirement equals the maximum profit that can be achieved. In that case the Lagrange multiplier of the profit constraint tends to infinity. Observe that the required profit may be the amount necessary to cover the operator's deficit in other markets (letters, etc.).

${ }^{3} \mathrm{We}$ implicitly assume that all the items bought by a consumer are subject to a single delivery. Consequently, the utility loss due to the fact that the delivery mode differs from the consumers preferred one does not depend on $x$.

${ }^{4}$ The pass-through rate could be less than one or even exceed one under imperfect competition. Because we assume that the pass through rate is 1 , we could treat $\mathrm{p}$ and $\mathrm{q}$ as equivalent. However, we maintain the separate notation to highlight the difference between the prices delivery companies charge to e-commerce retailers and the effect of those prices on what consumers pay.

${ }^{5}$ This is a typical result in second-best models, where the presence of a distortion (prices differing from marginal costs) may make it efficient to introduce additional distortions.

${ }^{6}$ In reality, this aspect probably reduces to the location of the relay closest (most conveniently located) for a given customer. We collapse all this heterogeneity into a single dimension.

${ }^{7}$ The demands are independent because the market segments covered by variety 0 and by variety 1 do not touch. They are separated by operator E's market. Recall also that we are characterizing a Nash equilibrium; operator $P$ is not a Stackelberg leader.

${ }^{8}$ These expressions are relatively simple because all the terms pertaining to the marginal consumers cancel out (because the marginal consumer has by definition the same utility with the two relevant modes of delivery).

${ }^{9}$ Alternatively, we could impose $\bar{\pi}=\mathbf{0}$ throughout and vary the amount of fixed costs, but this would make the comparison of welfare levels more cumbersome.

${ }^{10}$ When $\alpha=3$, the market share of home delivery is now $42 \%$ when $40 \%$ more expensive and $90 \%$ when relay delivery is $18 \%$ more expensive.

${ }^{11} 245$ corresponds to the maximum profit the postal operator can achieve in duopoly

${ }^{12}$ This result (due to the strategic interactions between firms) may seem counterintuitive at first sight but is reminiscent of many such results found in the mixed oligopoly literature (which studies imperfect competition between firms differing in their objective); see for instance Cremer et al. (1991). Such a result could of course not arise if operator $P$ were a Stackelberg leader. Recall that we study the Nash equilibrium.

${ }^{13}$ Many of these findings are based on the simulations and have to be qualified accordingly.
} 